Published in Langages, 2021, vol. 2, no. 222, pp. 77-94, which should be cited to refer to this work.

\title{
La compétence interculturelle et l'humour comme stratégie dans le contexte du management international
}

\section{Intercultural Competence and the Use of Humor in the International Management Context}

\author{
Pia Stalder \\ Haute Ecole d'Ingénierie et de Gestion du Canton de Vaud, Suisse (HEIG-VD, \\ HES-SO) \\ Christian Agbobli \\ Université du Québec à Montréal, Canada (UQAM)
}

\begin{abstract}
Résumé
Cet article s'inscrit au carrefour de la sociologie, de l'anthropologie, de la linguistique et de la pragmatique interculturelle. II porte sur les compétences interculturelles et, plus particulièrement, sur l'humour comme stratégie de communication en milieu professionnel international. D'une part, notre objectif est de donner un aperçu des possibilités et des limites de l'humour dans les interactions entre personnes d'origines et d'appartenances différentes. D'autre part, nous nous intéressons aux compétences clés de managers - femmes et hommes - qui travaillent dans des contextes sociaux complexes et ne peuvent, souvent, compter sur une histoire collective.

Nous présenterons d'abord un état des lieux sur l'humour dans le management international. Puis, sur la base d'une étude de cas issue d'un projet de recherche interdisciplinaire et empirique, nous examinerons les fonctions et les effets de l'humour dans les interactions avant d'aborder la question des compétences de communication nécessaires pour travailler sur les scènes internationales.
\end{abstract}

Mots-clés

communication, interculturel, compétence, humour, management

\begin{abstract}
This article falls within sociology, anthropology, linguistics and intercultural pragmatics. It is focused on intercultural competences and, more specifically, on humor as a communication strategy in the international workplace. Our goal is to provide an insight into the advantages and limits of humor in interactions between people of different origins and affiliations. In this sense, the article aims at reflecting on the key skills of managers - women and men - who work in complex social contexts: depending on their collective history, they cannot count on solid common communication ground.

We will first present an inventory of research papers on the subject of humor in international management. Then, based on a case study from an empirical research project, we will examine the functions and effects of humor during the verbal and non verbal interactions of a
\end{abstract}


multicultural team. In the end we will address the question of the communication skills that are needed for working on the international stage.

\section{Keywords}

Communication, Intercultural, Competence, Humour, Management

\section{INTRODUCTION}

Dans le monde professionnel contemporain, tout manager ${ }^{1}$ ou responsable de projet est potentiellement amené à travailler à l'échelle internationale. Il doit nécessairement chercher, développer et soigner la collaboration avec des personnes de diverses origines et appartenances ${ }^{2}$ qui vivent, voient, interprètent et disent le monde - et surtout les relations sociales - de façons différentes. La prise en compte de cette diversité dans la communication et les interactions au travail constitue un enjeu majeur pour les entreprises et les collaborateurs. Dans ce sens, plusieurs chercheurs ont soulevé l'importance de développer des compétences spécifiques (p. ex. Spitzberg 1991, Chen \& Starosta 1996, Bennett 2015 ou Koester \& Lustig 2015).

Nous considérons les compétences de communication interculturelle comme le fondement de la cohabitation, de la collaboration et de la compréhension entre les individus. Ces compétences vont au-delà des compétences linguistiques et sociolinguistiques ; d'après Gohard-Radenkovic (1999), elles impliquent également la maîtrise des composantes socioculturelles, discursives, sémiotiques et pragmatiques. Dans cet article, nous proposons d'analyser l'humour en tant que composante des compétences de communication interculturelle à acquérir dans un contexte de management international.

Dans un premier temps, nous donnerons un aperçu des fondements théoriques sur lesquels nous basons nos réflexions et analyses, notamment les notions de communication, de management et de compétence. Nous nous intéresserons ensuite à l'humour, à ses fonctions et à ses effets dans le contexte spécifique du management international, en nous inspirant d'études de cas issues d'un projet de recherche interdisciplinaire et empirique. Notre but est de mieux appréhender les possibilités et les limites de l'humour comme stratégie sur des terrains de communication complexes qui ne sont pas consolidés. Notre perspective est celle de la pragmatique interculturelle : interagir sur la scène internationale va de pair avec la négociation et la co-construction de «terrains communs » (cf. Kecskes 2013 et ce volume, Baider \& Cislaru, ce volume). Pour conclure, nous réfléchirons aux compétences nécessaires pour la co-construction de ces terrains, autrement dit, de cultures de collaboration, en milieu professionnel international.

\section{L'HUMOUR DANS LE MANAGEMENT INTERNATIONAL}

\footnotetext{
${ }^{1}$ Dans cet article, les termes employés pour désigner des personnes sont pris au sens générique; ils ont à la fois valeur de féminin et de masculin.

${ }^{2}$ La notion d'appartenance est entendue ici en termes sociologiques (cf. p. ex. Maalouf 1998). Chaque individu a plusieurs groupes d'appartenance (p. ex. famille, village, ville, région, nation, groupe professionnel, équipe sportive, parti politique, etc...). Le terme ne se limite donc pas à l'appartenance nationale d'une personne.
} 


\subsection{Communication, compétence et management interculturels}

Dans cet article, nous entendons par communication interculturelle l'importance des modalités de communication entre individus porteurs de cultures différentes ${ }^{3}$, le rôle de la relation interpersonnelle ainsi que les enjeux de pouvoir qui les caractérisent (Neuliep 2009, Stoiciu 2011, Bourassa-Dansereau \& Yoon 2017). Selon Stoiciu et Hsab (2011 : 10) «l'interculturel désigne souvent une rencontre, une relation de coprésence culturelle entre individus ou groupes, acteurs de la communication ». Comme le signalent Baider \& Cislaru (ce volume), toute communication repose, en effet, «non seulement sur les normes qui émergent au cours de la situation de communication, mais également sur des normes et des modèles culturels spécifiques aux communautés linguistiques et culturelles auxquelles appartiennent les interlocuteurs ». L'un des enjeux de la communication interculturelle repose sur la rencontre avec l'autre et sur les «problèmes » de communication possibles. Les notions qui y sont associées sont entre autres l'interaction, la définition de la situation et la perception. Agbobli et Kane (2014:334) soulignent que

pour les communicologues, l'interculturel est avant tout un enjeu d'interactions et d'échanges qui nécessite l'étude des conditions qui rendent la communication possible dans un contexte particulier.

Il est néanmoins difficile d'aborder la communication interculturelle sans se référer à la communication internationale, « son miroir » (Stoiciu \& Hsab 2011). En effet, la communication interulturelle a souvent été associée à un processus interpersonnel alors que la communication internationale est présentée comme relevant d'enjeux plutôt macro. Or, ces deux types de communication ne peuvent être séparés l'un de l'autre. Comme le soulignent Stoiciu et Hsab (2011 : 10)

les communcations internationales servent de contexte pour comprendre les enjeux d'interculturalité, alors que les communications interculturelles servent de contextes pour comprendre les enjeux d'internationalité, pour ainsi dire.

Il nous importe également de rester attentifs à la distinction entre « management interculturel » et «management international » (Mutabazi \& Pierre 2008, Baldauf \& Lang 2016, Bolten 2017). La notion de management interculturel englobe les processus de questionnement, de développement et d'organisation de formes de coopération entre individus d'origines et d'appartenances différentes qui sont amenés à collaborer dans des espaces - géographiques, politiques, économiques, historiques et surtout socioculturels - hétérogènes et fort complexes.

Le management international désigne, quant à lui, des structures de gestion et de direction, structures qui portent toutes les activités intra- et transnationales d'une entreprise avec la prise en compte des conditions-cadres spécifiques (géographiques, politiques, économiques et juridiques). À l'instar du rapport entre la communication

\footnotetext{
3 Selon plusieurs chercheurs (p. ex. Hall 1959 ou Ladmiral \& Lipianski 1989), les individus ne représentent pas leur culture. On ne peut trouver chez chaque personne l'entièreté des valeurs, des traits et des caractéristiques de sa culture; toutefois cette personne dispose de certains éléments de sa culture. Ce faisant, les individus portent des éléments caractéristiques de leur culture, ce sont des porteurs de culture.
} 
internationale et la communication interculturelle tel que nous venons de l'exposer au début de cette partie, le management international et le management interculturel sont étroitement liés : la définition d'une stratégie internationale et le succès de sa mise en pratique dépendent essentiellement des compétences interculturelles de tous les managers, équipes et acteurs sociaux concernés.

Quant à la notion de compétence interculturelle, elle intéresse les sciences de gestion depuis les années 1950 déjà (Bolten 2017 : 188). Bolten (op. cit.) différencie deux courants de recherche. Le premier, celui des «modèles de listes et de structures », est le plus ancien (p. ex. Lysgaards 1955 ou Gardner 1965). Il se caractérise par des modes de pensée et de conception en termes de «structures » et de « classes ». Les travaux de recherche visent l'identification de traits personnels de managers expatriés et l'élaboration de typologies. La compétence est appréhendée ici sous forme d' "addition» de composantes distinctes. Dans le deuxième courant par contre, le courant des « modèles de processus », la compétence interculturelle est conceptualisée comme «construction multiple » et dynamique (Rauner 2004 : 8, cf. Bolten op. cit. : 189). Les modèles de ce courant-ci se basent sur une définition de la notion de culture comme forme d'organisation négociée par les individus dans leurs interactions au quotidien (Wicker 1997, Rivera 2000, Giordano 2003 ou GohardRadenkovic \& Stalder 2013). La compétence interculturelle est considérée comme «conjugaison» de compétences personnelles, sociales, professionnelles et méthodologiques (cf. p. ex. Erpenbeck 2001 ou Mahadevan \& Kilian-Yasin 2013). Nous adhérons à cette vision-ci de la compétence interculturelle. Il s'agit d'une vision plus contemporaine. Elle est également en phase avec le point de vue de Le Boterf $(2010: 68)$ :

Résultant d'un savoir agir, d'un vouloir agir et d'un pouvoir agir, la production d'une action compétente relève d'une responsabilité partagée entre la personne elle-même, le management, le contexte de travail (organisation du travail, conditions de travail, moyens, système de classification et de rémunération...) [...].

Il existe une abondance de recherches et de publications sur la communication, le management et la compétence interculturels ( $c f$. p. ex. Ladmiral \& Lipiansky op. cit., Chen \& Starosta 1996, Mutabazi \& Pierre 2008, Barmeyer \& Franklin 2016, Bolten, op. cit., Meier 2019 ou encore la série Transversales ${ }^{4}$ pour des synopsis).

Ces trois domaines, quand bien même distincts, sont congruents sous divers angles, notamment en ce qui concerne la dimension épistémique. En effet, la perspective interculturelle en communication, en management et en compétence est souvent abordée selon une dimension qualitative ou quantitative. Par exemple, dans le domaine du management interculturel, les travaux se basent soit sur des données quantitatives et des approches cross-culturelles généralisantes (Hofstede 1987 ou 2001, Trompenaars 1994), soit sur des données qualitatives analysées de façon fine et précise (Chevrier 2003, d'Iribarne 1989, Chanlat 1990, Mutabazi \& Pierre op. cit., Stalder 2010 ou 2019). Dans nos travaux de recherche - au carrefour de

\footnotetext{
${ }^{4}$ La collection Transversales (éditions Peter Lang) s'intéresse aux intersections possibles entre langues, sociétés et cultures : https://www.peterlang.com/abstract/serial/TRANSV?rskey=JTuA2N\&result=1 (consulté le 25.2.20).
} 
l'anthropologie, de la sociologie, de la linguistique et de la pragmatique interculturelle - portant sur les interactions en milieu professionnel international nous privilégions l'approche inductive et qualitative. L'étude de cas qui se trouve au cœur de cet article en est un exemple.

\subsection{L'humour, un mode de communication entre art, émotion et stratégie}

\subsubsection{Etat des lieux sur l'humour}

L'humour et le rire ne sont pas des terrains de recherche nouveaux. Il existe, en effet, de nombreuses recherches sur l'humour comme pratique sociale, mode de communication ou stratégie de management, sur ses fonctions, ses effets, ses avantages et ses risques (voir Defays 1996 ou 1999, Martin 2003 ; Martin, Rich \& Gayle 2004 ; Romero \& Cruthirds 2006 ; Charaudeau 2006; Bottega 2008 ; Lang \& Lee 2010; Wood \& al. 2011; Autissier \& Arnéguy 2012; Wisse \& Rietzschel 2014 ; Chlopicki \& Brzozowska 2017). Bien que pour Avolio et al. (1999) l'humour augmente la productivité, la recherche montre qu'il y a différents types d'humour qui ont des effets positifs ou négatifs dans l'interaction. Les résultats de recherche de Lang \& Lee (op . cit.) montrent par exemple qu'un humour qui désinhibe a un impact positif sur la créativité dans un groupe, tandis qu'un humour inhibiteur provoque l'effet contraire.

Cependant, à notre connaissance, il n'y a que très peu de recherches sur l'humour, sur ses possibilités et sur ses limites dans le contexte particulier du management international et interculturel (cf. aussi Pluta 2014, 2015 ou Pluta \& Höber 2017). Plusieurs auteurs font allusion aux enjeux interculturels dans l'usage de l'humour en milieu professionnel. Or, ils restent dans un discours culturalisant, c'est-à-dire dans une vision des cultures comme ensembles de traits distinctifs, statiques et fermés (Clouse \& Spurgeon 1995, Romero \& Cruthirds 2010, Autissier \& Arnéguy 2012, Tricart 2015). Les recherches qui abordent l'humour dans une perspective véritablement interculturelle (Grindsted 1997, Holmes 2000, Pullin 2007, Stalder 2010 et 2019), c'est-à-dire dans une perspective basée sur une conception ouverte et dynamique de la notion de culture comme forme d'organisation co-construite par les individus dans leurs interactions au quotidien (Gohard-Radenkovic \& Stalder, op. cit.), sont encore trop rares.

L'originalité de notre approche au sujet de l'humour dans le management international et interculturel réside d'une part dans notre cadre théorique et dans notre méthode de recherche qui sont les deux interdisciplinaires (sociologie ${ }^{5}$, anthropologie $^{6}$, psychologie ${ }^{7}$, linguistique et pragmatique interculturelle ${ }^{8}$ ). D'autre part, nos analyses portent sur des données «authentiques », c'est-à-dire sur des interactions et séquence d'humour observées et filmées dans des entreprises et organisations internationales différentes.

\footnotetext{
${ }^{5}$ Voir Goffman (1973a, 1973b, 1974, 1981 ou 2005) ; Bourdieu 1980 ou 1982 ; cf. également TingToomey \& Kurogi 1998 ou Merkin 2018.

${ }^{6}$ Voir Geertz 1973 ; Gallissot, Kilani \& Rivera 2000 ; Winkin 2001 ou Kilani 2009.

${ }^{7}$ Voir p. ex. Moscovici 1986 ou Jodelet 1997.

${ }^{8}$ Cf. Kerbrat-Orecchioni 1990, 1992 ou 2000; House 2002a, 2002b; House \& Rehbein 2004 ou Mondada 2005.
} 


\subsubsection{Définitions de l'humour}

Dans notre article, nous employons le terme « humour » pour désigner une notion générique. L'humour est un mode de communication universel (Apte 1985, Lefcourt 2001). Martin (2007: 5) le définit ainsi :

[humor] refers to anything that people say or do that is perceived as funny and tends to make others laugh, as well as the mental processes that go into both creating and perceiving such an amusing stimulus, and also the affective response involved in the enjoyment of it. ${ }^{9}$

\section{D'un point de vue psychologique, l'humour est considéré comme}

une forme particulière d'émotion positive caractérisée par un ressenti d'amusement, de plaisir, exprimé par le sourire ou le rire, qui met en jeu divers processus cognitifs et dont les différentes modalités sont déterminées par le contexte personnel et social (Derouesné 2016 : 95).

Dans la littérature examinée, l'humour est également décrit comme un art de communication et un outil de séduction ou de manipulation. Pour Morreall (1989) l'humour est «le plaisir de la bizarrerie ». Fry (cité dans Wittezaele \& Garcia 2002) le considère comme un « rafraîchissement mental» tandis que Bottega (2008) parle d'un «levier de changement» ou encore Tricart (2015) d'un «formidable antidépresseur $\gg$.

L'humour s'inscrit dans une « relation triadique » (Charaudeau, op. cit. : p. 2-3). Il met en scène trois protagonistes :

- le locuteur,

- le destinataire et

- la cible.

Le locuteur est celui qui produit l'acte humoristique dans une situation d'interaction donnée. Charaudeau décrit le destinataire comme «témoin de l'acte humoristique ». Il peut être complice - et ainsi « appelé à entrer en connivence avec le locuteur »- ou victime. Dans ce cas-ci, «il est à la fois destinataire et cible de l'acte humoristique » qui peut se sentir agressé. La cible est «ce sur quoi porte l'acte humoristique ou ce à propos de quoi il s'exerce » (ibid.). L'acte humoristique peut, entre autres, porter sur

- le locuteur soi-même (autodérision),

- les semblables,

- les autres,

- des situations ou des choses.

\footnotetext{
${ }^{9}$ Traduction en français : « [1'humour] désigne tout ce que les gens disent ou font qui est perçu comme drôle et qui tend à faire rire les autres, ainsi que les processus mentaux qui entrent en jeu à la fois dans la création et dans la perception d'un tel stimulus amusant, et aussi la réponse affective qu'implique le fait d'en profiter».
} 
Defays (1996: 8-9), quant à lui, souligne que l'humour joue souvent avec les contraires : il porte fréquemment sur le «bien» contrasté au «mal». D'après cet auteur, l'humour (noir) donne - sous couvert de l'implicite - l'occasion de « faire ou de dire du mal sans s'exposer à des reproches, mais en suscitant au contraire les encouragements des rieurs » (ibid.: 11). De plus, l'auteur met en avant que l'humour permet à l'individu de triompher du malheur et de la malveillance, en les réduisant à des inconvénients dérisoires ${ }^{10}$.

Pour ce qui est du rire, celui-ci est l'effet physiologique d'une tension. Il a une valeur communicative ( $c f$. Defays, op. cit. ou Adelswärd, 1987) et donc des significations ainsi que des fonctions sociales ( $c f$. Bergson 1900/1917).

Le rire est soit unilatéral (rire de), soit partagé (rire avec). En d'autres termes, les rires peuvent être exclusifs ou inclusifs. D'après Adelswärd (op. cit.), le rire accomplit des objectifs interactionnels complexes. Ses fonctions sont multiples: s'excuser pour son ignorance, marquer un comportement auto-ironique ou une attitude de modestie, atténuer un constat, etc. Le rire est à même de s'ajouter ou de se superposer au discours et d'inviter l'autre à le rejoindre, donc à adopter la même perspective. Il a le pouvoir de «faire le pont» (Grindsted 1997), autrement dit de resserrer ou de construire des groupes et de réguler le climat interactionnel.

En effet, l'humour et le rire revêtent de multiples formes et fonctions. Ils sont inextricablement liés à leur contexte d'émergence. L'humour et le rire sont insoupçonnables, imprévisibles ; ce d'autant plus que leur appréciation s'inscrit dans l'interaction et la relation entre émetteur(s) et récepteur(s). Qui le dirait mieux que William Shakespeare : «A jest's prosperity lies in the ear of him that hears it, never in the tongue of him that makes it. » (1598, Love's Labour's Lost, V, 2).

Or, l'humour ne se mesure pas au rire. Celui-ci n'est point «garant du fait humoristique » (Charaudeau $2006: 1$ ). Car, «si le rire a besoin d'être déclenché par un fait humoristique, celui-ci ne déclenche pas nécessairement le rire » (ibid.). Charaudeau souligne à juste titre qu'il faut encore que l'humour soit perçu comme tel, ce qui est d'autant plus difficile que les personnes ont des appartenances, des valeurs, des histoires et des expériences de vie différentes.

Il s'ensuit de cet état des lieux que l'humour revêt de forts potentiels stratégiques. En effet, nos propres recherches sur la communication internationale et interculturelle (Stalder 2010, 2013 \& 2018 et Agbobli \& Hsab 2011, Agbobli \& Kane 2014, Agbobli 2018) montrent que l'humour ne revêt pas seulement les caractéristiques que nous venons d'exposer. L'étude de cas qui est présentée et discutée dans la partie suivante a pour but d'ouvrir la perspective sur les fonctions stratégiques de l'humour, ses avantages et ses limites dans le contexte spécifique du managment international.

\subsection{Fonctions et effets de l'humour : présentation d'une étude de cas}

Travailler, c'est du sérieux. Mais, pas tout le temps. C'est un de nos constats dans le cadre de nos projets de recherche empirique sur les interactions verbales et non verbales entre personnes d'origines et d'appartenances différentes (Stalder

${ }^{10}$ Et Defays ajoute: « En ce sens, l'expression ‘humour noir' serait pléonastique ». 
2010, 2012, 2014a, 2014b, 2016a, 2016b, 2019a, 2019b). Les exemples présentés ici proviennent d'un projet - clos en 2010 - qui avait pour objectif d'identifier les stratégies de communication - verbales et non verbales - dans les réunions en contexte professionnel international (cf. Stalder 2010). En effet, les participants des séances observées recouraient bien souvent à l'humour. De la sorte, nous l'avons étudié de plus près.

Dans un premier temps, nous avons passé en revue nos 400 pages de notes d'observation prises pendant notre travail sur le terrain afin de repérer les occurrences de séquences d'humour et de rire. En voici un exemple :

Figure 1. PharmaPunkt, journal de terrain 1, p. 7

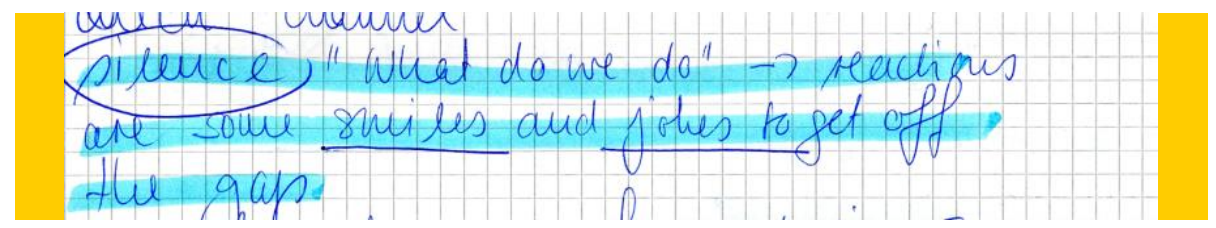

Nos notes d'observation - ainsi que les autres types de données récoltées proviennent de trois terrains différents. Nous avons observé d'une part les interactions dans les réunions au sein de deux entreprises pharmaceutiques (sises à Bâle, en Suisse $)^{11}$ et d'une organisation humanitaire (sise à Genève) $)^{12}$. D'autre part, ces réunions ont été filmées (12 heures de film en total, au moins 3 heures par terrain). De plus, près de 50 entretiens semi-directifs ont été menés avec les participants aux réunions observées ${ }^{13}$.

Suite à l'analyse des journaux de terrain, nous avons identifié dans les entretiens et dans les films - cela en suivant la théorie et méthode du «Rich Point 》 (Agar 1994) - les séquences-clés en termes d'humour. Ces séquences ont été transcrites in extenso. Puis nous avons procédé à une analyse de discours pour les entretiens et à une analyse interactionnelle pour les extraits filmiques. Pour ce faire, nous nous sommes notamment basés sur les travaux de Moeschler (1985), Gülich (1986), Gülich \& Kotschi (1987), Kerbrat-Orecchioni (1989, 1990, 1992, 1996, 1998a, 1998b ou 2000), Mondada (2005), Stalder (2010 ou 2019) ${ }^{14}$.

Nos résultats montrent que l'humour compte parmi les stratégies de communication empruntées par les participants aux réunions en milieu professionnel international. L'humour émerge des interactions. Il revêt différentes formes et

\footnotetext{
${ }^{11}$ Pseudonymes : Formalis et PharmaPunkt.

${ }^{12}$ Pseudonyme : Humanitas.

13 À l'époque des enquêtes sur ces terrains (entre 2000 et 2005) Formalis employait plus de 74000 collaboratrices et collaborateurs dans le monde. À Bâle, lieu de récolte des données, travaillaient plus de 6000 personnes de 85 nationalités différentes. PharmaPunkt, quant à elle, employait plus de 65000 personnes dans le monde, dont plus de 7000 en Suisse. À Bâle travaillent plus de 5000 personnes de 56 nationalités différentes. Pour ce qui est d'Humanitas, l'organisation disposait à l'époque de délégations dans 60 pays du monde, déployait des activités dans plus de 80 pays et employait environ 12500 personnes. À Genève travaillaient 820 personnes de 52 nationalités différentes.

${ }^{14}$ Pour le détail des données et de la méthodologie cf. Stalder (2010).
} 
fonctions. Les effets de ce jeu sont fortement liés aux appartenances et expériences individuelles d'une part, aux relations et à l'histoire du groupe d'autre part.

Par la suite, nous présenterons des séquences interactionnelles représentatives de nos observations. Dans le but de resserer la portée réflexive du texte, mais surtout afin de nous permettre de procéder à une «description dense »-thick description selon le bon mot de Geertz (1973) - d'une situation concrète qui met en scène l'humour dans un contexte de management international, nous nous limitons à un seul exemple dans cet article. Toutefois, nous le commenterons en détail - pour d'autres « images de la pratique » voir Stalder (2010, 2019a ou 2019b). L'exemple en question est tiré d'une réunion filmée au sein de l'industrie pharmaceutique (Formalis). La section d'entreprise dont est issu l'événement filmé s'occupe de l'emballage de médicaments. La séance observée s'inscrit dans un cycle régulier de réunions formelles. Ces rencontres ont lieu une fois par semaine et elles ont pour but la planification et le suivi des projets - de la conception de l'emballage jusqu'à l'expédition des médicaments - ainsi que la coordination des tâches imparties à différents (sous-)sections et collaborateurs. Outre ces fonctions de planning et de coordination, la réunion vise la résolution ou l'évitement de complications.

Le jour de l'observation, huit personnes participent à la réunion : Karl (chef de section et président de la séance, il est d'origine suisse-alémanique), Lou (coordinatrice d'origine française), Julia (packaging manager d'origine allemande), Laure (packaging manager d'origine française), Hans (responsable de la mise en emballage, il est d'origine suisse-alémanique), James et Adrian (collaborateurs d'origine anglaise) et Fernando (collaborateur d'origine suisse et espagnole).

Dans le premier extrait ci-après, Laure est en train de soumettre au groupe un prototype d'un récipient tubulaire de médicaments. Elle vient de montrer qu'une petite étiquette autocollante, pourvue du minimum d'informations nécessaires, trouve tout juste sa place sur cet emballage. Lors de sa présentation, elle rit. Ce rire est probablement le signal d'une tension liée à un risque de perte de face ${ }^{15}$. Nous l'interprétons comme un rire d'embarras - parce que, en effet, l'étiquette et les caractères d'impression sont minuscules. Voici la séquence qui suit sa présentation $^{16}$ :

01 Karl: It fits, ja. May I have a look? ((prend l'emballage tubulaire dans les mains))

02 James: Probably half of it fits. ((rit))

03 Karl: *You need very strong glasses* to read this. ((se penche sur l'emballage))

04 Laure: Er *it's a, erm, it's a size six*, hein, *it's* ((rit, gestes: d'abord index pour

${ }^{15}$ Le rire représente ici ce que Gumperz (1982) appelle «a contextualisation cue».
${ }^{16}$ Conventions de transcription :
\begin{tabular}{ll}
\hline$\ldots]$ & omission \\
\hline$(($ description $))$ & «didascalies»: description de l'interaction \\
\hline\lceil & chevauchement L1 (sa parole coïncide avec celle de L2) \\
\hline$\bigsqcup\rfloor$ & chevauchement L2 (sa parole coïncide avec celle de L1) \\
\hline *expressions* & délimitation d'une action décrite
\end{tabular} 
$\mathbf{0 5}$ «attention», ensuite: bras étendus, paumes des mains tournées vers le bas et mues horizontalement

06 en entre-croisant les bras afin d'exprimer l'idée de «ça correspond juste à la norme»))

$07\lceil$ it's the limit $\rceil$.

08 Karl: $\lfloor *$ No, no* I am $\rfloor$ just joking ((gestes: réaction à la gestuelle de Laure, lève la main -

09 la paume vers l'intérieur - en protection de face)), maybe my glasses are not strong

10 enough anymore. No, it's okay. That's good, yes. ((passe l'objet à Lou))

Les quatre arrêts sur image - rendus anonymes pour des raisons de confidentialité - ont pour but de faciliter la lecture des descriptions de la gestualité dans les lignes 4 à 6 et 8 à 9 . Karl se trouve tout à gauche sur les images (au milieu on voit Julia) et Laure tout à droite.

Arrêts 1-2: gestes syllinguistiques ${ }^{17}$ de Laure accentuant l'idée «la norme est respectée».
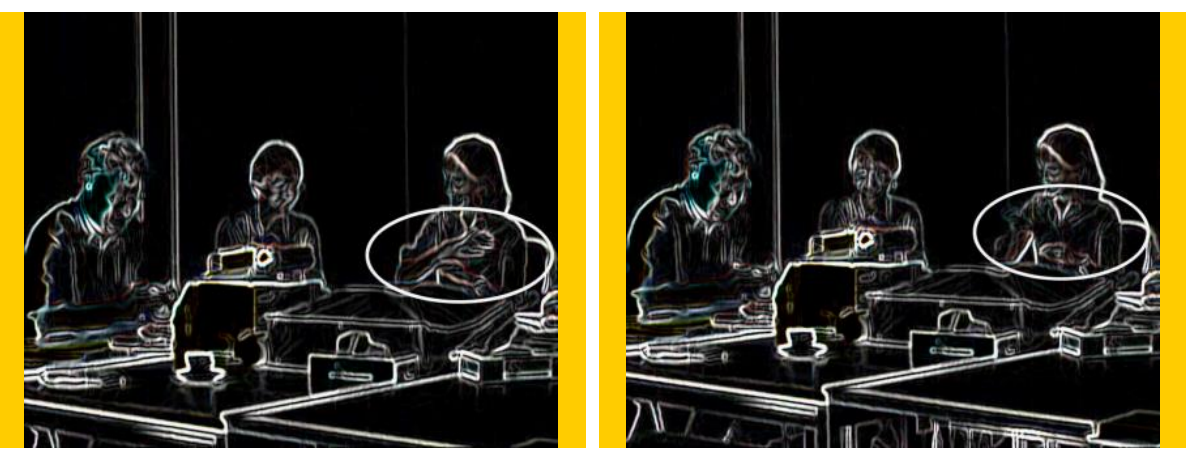

Arrêts sur image 3-4: Karl atténue de façon syllinguistique la menace de face contre Laure.

\footnotetext{
${ }^{17}$ Les gestes syllinguistiques sont des gestes communicatifs qui coexistent avec le langage parlé (cf. système de classification des gestes de Cosnier 1982).
} 

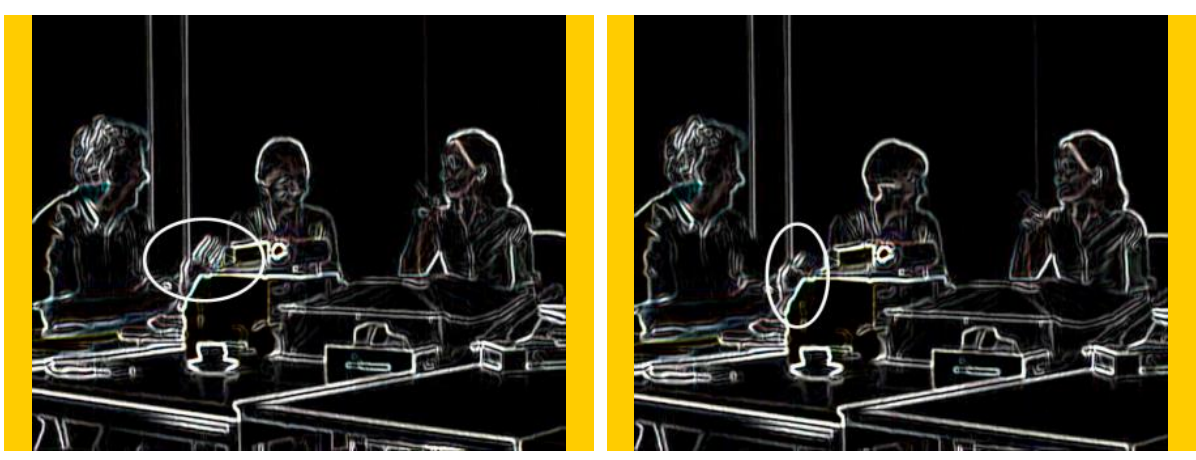

Les interactions dans cette équipe et au cours de cette réunion sont multilingues ( $c f$. p. ex. ligne 1 «ja ») : les participants passent d'une langue à l'autre, cela en fonction de la situation, des compétences linguistiques individuelles et de celles des destinataires premiers des informations échangées.

Karl a écouté Laure en observant attentivement sa démonstration. À la première ligne, il confirme qu'il l'a bien suivie. Le changement de langue qu'il opère est signe d'emphase (tout autant que le «hein » français de Laure à la ligne 4). Or il souhaite voir l'objet et l'étiquette de plus près. Le climat interactionnel - instauré par Laure elle-même - étant propice à des rires, James intervient avec une remarque ironique quand l'emballage passe des mains de Laure à celles de Karl (ligne 2). Pour Defays (1996) l'ironie est un des procédés comiques primaires qui fonctionne par des opérations de «remplacement», telles que l'euphémisme, l'antiphrase ou la (pseudo-)simulation. L'ironie contredit fréquemment la maxime de qualité ${ }^{18}$, donc il s'agit d'une affirmation contraire à la vérité. Tel est le cas de l'intervention de James. Il avance que, probablement, ce n'est que la moitié de l'information qui y figure: « Probably half of it fits. » et il auto-initie un rire qui donne le ton. ${ }^{19}$

Par la suite, Karl entre également sur le terrain du comique. Il utilise l'humour comme stratégie d'atténuation. L'humour contrevient souvent à la maxime de quantité (Grice 1979) et oblige le récepteur à tenter de trouver l'information qu'on ne veut pas lui dévoiler. Karl, moyennant la plaisanterie «you need very strong glasses to read this » (ligne 3 ), en français « il vous faut des lunettes très fortes pour lire ça », amortit son attitude critique face au projet d'emballage proposé par Laure. Toutefois, cette stratégie d'atténuation ne réussit que partiellement, puisque Laure (lignes 4-6) commence à se justifier immédiatement en soulignant qu'elle est dans la règle, vu que, dans le cas de son projet, il s'agit d'une « étiquette de taille six ».

Même si le climat interactionnel est toujours propice au rire - ce qui en soi détend la situation - on peut voir dans l'argument de Laure une menace de face envers Karl. Ainsi, ligne 8, celui-ci utilise d'abord une stratégie métadiscursive (cf. Kotschi 1986 ou Gülich 1986a), "No, no I'm just joking », accompagnée d'une gestualité syllinguistique (cf. arrêts sur image 3-4) et renforce sa réaction par une

\footnotetext{
${ }^{18}$ La maxime de qualité (Grice 1979) veut que le discours soit véridique et le mensonge évité.

${ }^{19}$ D'après Goffman (1974), il s'agirait ici de «keying, framing laughter», c'est-à-dire un rire qui cadre l'intervention de James et rend plus explicite son intention comique.
} 
remarque auto-ironique qui fonctionne comme stratégie auto-protectrice et hétéroprotectrice (cf. Stalder 2010) en même temps: "Maybe my glasses are not strong enough anymore » (en français «peut-être mes lunettes ne sont plus suffisamment fortes ») (lignes 9 à 10). ${ }^{20}$

La dynamique humoristique manifeste dans la séquence favorise la poursuite de l'examen de Karl. Il continue à évaluer le travail de Laure, tout en protégeant sa face et en ménageant celle de sa collaboratrice. In fine, il approuve le projet d'emballage présenté (ligne 10).

Connaissant la structure organisationnelle de ce département (nous avons étudié son organigramme et mené des entretiens avec tous les membres de l'équipe) et sachant, ainsi, que James est le supérieur hiérarchique de Laure, nous pouvons interpréter l'intervention de ce Group Head de plusieurs façons : comme acte de distanciation critique - en d'autres termes, de critique indirecte - ou, éventuellement, comme acte de soutien de sa subordonnée. Cette deuxième interprétation est soutenue par l'observation suivante: plus loin dans l'enregistrement filmique de cette réunion (alors que la langue d'interaction est passée de l'anglais à l'allemand pour permettre la participation active d'un membre de l'équipe, Hans, qui ne maîtrise pas suffisamment bien l'anglais), James agit de manière similaire. Il lance des propos humoristiques envers son collègue et compatriote Adrian qui se trouve dans un rapport tendu avec Hans, Karl et Lou. Voici ce deuxième extrait ${ }^{21}$ :

01 Lou ((à Adrian)): *Siehst du warum wir eigentlich den Namen

02 des Produktes* eingeben müssen und das Produkt nicht Blistering nennen?

$\mathbf{0 3}$ ((sourit, Adrian fait de même; elle se tourne ensuite vers les autres et dit, en riant :))

04 Sonst finden wir es nämlich nicht.

05 James ((en riant, à Adrian)): Die Studien ((sic)) heisst nicht

06 « Blistering »! ((ils rient tous))

Dans les deux extraits mis en évidence dans ce chapitre, l'humour se laisserait donc également lire comme stratégie d'évitement de conflits ou comme stratégie de (re)médiation, puisque James renforce le caractère détendu du climat interactionnel dans la réunion et contribue à la prévention de tensions et de stress dans le groupe. Ainsi, James - et l'humour qu'il utilise - opèrent comme médiateurs et modérateurs.

Comme nous l'avons souligné plus haut, l'utilisation et l'appréciation de l'humour sont fortement liés aux identités individuelles des membres d'un groupe et, en même temps, à leur histoire collective. Dans les exemples exposés, plusieurs conditions sont réunies qui favorisent les fonctions et effets positifs de l'humour. D'abord, les participants ont une histoire commune. Ils travaillent ensemble depuis plusieurs années déjà et ont eu le temps de co-construire leurs terrains de communication (cf. Kecskes ce volume). Ils se connaissent bien. Dans l'entretien,

\footnotetext{
${ }^{20}$ Sa remarque auto-ironique est proche du comportement humoristique «des pays au nord», tels que le décrivent Geoffroy (2001) et Grindsted (op. cit.).

${ }^{21}$ Pour faciliter la compréhension de ce deuxième extrait, nous proposons une traduction de l'interaction verbale. Lou : Vois-tu pourquoi nous devons entrer, au fait, le nom du produit et n'appelons pas le produit « Blistering »? Sinon, nous ne le trouverons pas. James : Les études ne s'appellent pas « Blistering ».
} 
Adrian a avancé par exemple que «people have a fairly close day to day contact». Ensuite Laure, quand elle présente son travail, est consciente du fait qu'avec son projet elle frôle les limites, même si elle a respecté les normes, d'où son rire d'embarras. L'intervention de James ne la surprend guère, elle entend l'humour comme tel et peut rejoindre le rire hétéro-initié de celui-ci sans se sentir menacée. Karl enfin déclare ouvertement qu'il plaisante. Tous ces aspects ont pour conséquence que l'humour et le rire qu'il provoque sont «compris » et n'excluent $a$ priori personne.

D'une part, ces extraits sont représentatifs du côté «émergent 》 de l'humour et de ses fonctions et effets multiples dans un contexte professionnel interculturel. Comme illustré, l'humour est utilisé comme stratégie d'atténuation, d'évitement de conflits ou de pertes de face, comme stratégie de médiation ou de modération. Jouer avec l'humour a des effets de distanciation. Il peut «dédramatiser » des situations ou des résultats frôlant les limites de l'acceptable.

D'autre part, les deux exemples illustrent la complexité du jeu interactionnel codéfini par les expériences et identités non seulement des individus, mais aussi des groupes et cultures qu'ils forment ensemble.

\section{Conclusion : UNE EPEE A DOUBLE TRANCHANT}

Dans notre article, nous nous sommes intéressées à l'humour comme stratégie de communication en milieu professionnel international où son émergence et son appréciation sont particulièrement dépendantes de l'histoire individuelle et collective des personnes en interaction. Car, la dimension interculturelle a un impact sur les actions et réactions: en particulier, sur la complexité des (en)jeux de figuration (cf. Goffman 1974, 2005 ou Merkin 2018). Les participants puisent leur créativité humoristique et l'appréciation de celle-ci dans tout ce que leur offre le moment interactionnel, dans tout ce qu'ils peuvent - et veulent - y voir.

En somme, l'humour est une épée à double tranchant. D'un côté, il a des fonctions et des effets positifs. Il peut être une source d'optimisme, de proximité plus forte en termes de relations sociales, d'amélioration de la communication et de la socialisation (voir aussi Guibert, Paquerot \& Roques 2002). L'humour permet de sauver la face et d'adoucir des messages déplaisants (voir aussi Lyttle 2007). De même, l'humour contribue à la réduction de stress.

De l'autre côté, l'humour est un mode d'expression particulier. Il ne se décrète pas, il émerge dans et de l'interaction. L'humour peut offenser, blesser, mettre mal à l'aise, exclure. Les risques de faux pas sont d'autant plus inhérents que des personnes d'origines différentes sont présentes. Afin d'atténuer les risques de l'humour dans la communication et le management interculturels en milieu international, plusieurs aspects méritent d'être pris en compte :

- le contexte et les participant-e-s dans toutes leurs complexités ;

- l'histoire du groupe : les participant-e-s ont-ils une histoire commune qui facilite la reconnaissance de l'humour en tant que tel ?

- l'accessibilité : l'humour est-il accessible pour tous, autrement dit, le passage du mode «sérieux» au mode «ludique» est-il suffisamment explicite et compréhensible pour les personnes présentes et concernées ? 
- l' "objectivité » : rire de situations ou d'objets peut, selon le contexte et l'histoire du groupe, être plus prudent que rire de personnes et de groupes ;

- l'inclusion versus l'exclusion : un rire avec (rire inclusif) est préférable au rire de (rire exclusif).

En conclusion, l'humour dans le management international et interculturel représente un défi particulier. Il exige de fortes compétences interculturelles, donc une capacité à conjuguer des compétences personnelles, sociales, professionnelles et méthodologiques. Les éléments clés de ces compétences sont à notre avis la bonne (auto-)observation, l'écoute et le métadiscours - trois «ingrédients de base » pour un humour partagé. 


\section{Références}

AgAR M. (1994), Language Shock. Understanding the Culture of Conversation, New York, Willam Morrow.

Agbobli Ch. (2018), « Mon dépanneur est vietnamien ou les stéréotypes à la rescousse de la communication interculturelle dans le contexte du Québec », Questions de communication 33(1), 169-186.

Agbobli Ch. \& Hsab G. (éds) (2011), Communication internationale et communication interculturelle: regards épistémologiques et espaces de pratique, Québec, Presses de l'Université du Québec.

Agbobli Ch. \& Kane O. (2014), «Communication interculturelle provoquée : entre identité et recadrage présentiel », in N. Corbu, D. Popescu-Jourdy \& T. Vlad (eds), Identity and Intercultural Communication, NewCastle upon Tyne, Cambridge Scholars Publishing, 330-348.

Avolio B. J., Howell J. M. \& SosiK J. J. (1999), “A funny thing happened on the way to the bottom line: Humor as a moderator of leadership style effects", Academy of Management Journal 42(2), 219-227.

BAider F. \& Cislaru G. (ce volume), Communication et intercompréhension: Regards croisés de la pragmatique interculturelle et de la pragmatique contrastive.

BALDAUF N. \& LANG R. (2016), Interkulturelles Management, Wiesbaden, Springer.

Barmeyer Ch. \& Franklin P. (2016), Intercultural Management. A Case-Based Approach to Achieving Complementarity and Synergy, London, Palgrave Macmillan.

BARMeYer, CH. \& Davoine, E. (2016), « Konstruktives interkulturelles Management - Von der Aushandlung zur Synergie. Constructive intercultural management - From negotiation to synergy », Interculture journal 15/26, 97-116.

Bergson H. (1900/1917 - 16E ÉD.), Le rire, Essai sur la signification du comique, Paris, Alcan.

Bolten J. (2018), Einführung in die Interkulturelle Wirtschaftskommunikation, Göttingen, Vandenhoeck \& Ruprecht.

BotTega C. (2008), «L'humour est-il un outil de management ? , Humanisme et Entreprise, 2008/3 ( $\mathrm{n}^{\circ}$ 288), 21-34, [Consulté le 08-01-2019, https://www.cairn.info/revuehumanisme-et-entreprise-2008-3-page-21.htm ; DOI 10.3917/hume.288.0021]

BOUgHeRra T. (2007), «Humour et didactique des langues : pour le développement d'une compétence esthético-ludico-référentielle », Études de linguistique appliquée 3 (147), 365-382.

BOURASSA-DANSEREAU C. \& YOON C. (2017), «Communication interpersonnelle et communication interculturelle : enjeux de leurs croisements », in C. Montgomery \& C. Bourassa-Dansereau (éds), Mobilités internationales et intervention interculturelle, Québec, Presses de l'Université du Québec, 31-46.

BouRdieu P. (1980), Le sens pratique, Paris, Les Éditons de Minuit.

Bourdieu P. (1982), Ce que parler veut dire. L'économie des échanges linguistiques, Paris, Fayard.

CAZAdE A. (2009), «L'interculturel est-il soluble dans l'humour? », Cahiers de l'APLIUT 28 (2), 24-39, [consulté le 8-01-2019, http://apliut.revues.org/1067 .

Charaudeau P. (2006), Des catégories pour l'humour, Revue Questions de communication 10, Nancy, Presses Universitaires de Nancy [consulté le 24-9-20, https://www.patrickcharaudeau.com/Des-categories-pour-l-humour.html]. 
Chen G.-M. \& Starosta W. J. (1996), « Intercultural Communication Competence: A Synthesis », Communication Yearbook 19, 353-383.

Chlopicki W. \& Brzozowska D. (2017), Humorous Discourse, Boston/Berlin, de Gruyter.

COSNIER J. (1982), Les voies du langage, Paris, Bordas.

CSIKSZENTMIHALYI M. (1996), Creativity: Flow and the psychology of discovery and invention, New York, Harper/Collins.

Defays J.-M. (1996), Le comique, Paris, Seuil.

Defays J.-M. \& Rosier L. (1999), Approches du discours comique, Dolembreux, Pierre Mardaga éditeur.

ERPENBECK J. (2001), «Wissensmanagement als Kompetenzmanagement», in G. Franke (Hrsg.), Komplexität und Kompetenz. Ausgewählte Fragen der Kompetenzforschung, Bielefeld, Bertelsmann, 102-120.

GALISSON R. (2002), «L'humour au service des valeurs : défi salutaire, ou risque inutile », Le français dans le monde $\mathrm{n}^{\circ}$ spécial, 122-139.

Gallissot R., Kilani M. \& Rivera A. (2000), L'imbroglio ethnique, Lausanne, Payot.

GeERTZ C. (1973), The Interpretation of Cultures. Selected Essays, New York, Basic Books.

GeOFFroy Ch. (2001), La mésentente cordiale. Voyage au cœur de l'espace interculturel franco-anglais, Paris, Éditions Grasset \& Fasquelle / Le Monde de l'Éducation.

GioRdano CH. (2003), « Préface. De la crise des représentations au triomphe des préfixes » in A. Gohard-Radenkovic, D. Mujawamariya \& S. Pérez (éds), Intégration des «minorités» et nouveaux espaces interculturels, Berne, Peter Lang, 11-17.

GOFFMAN E. (1973A), La mise en scène de la vie quotidienne. 1. La présentation de soi, Paris, Les Éditions de Minuit.

GofFMAN E. (1973B), La mise en scène de la vie quotidienne. 2. Les relations en public, Paris, Les Éditions de Minuit.

GoFFMAN E. (1974), Les rites d'interaction, Paris, Les Éditons de Minuit.

GOFFMAN E. (2005), Interaction Ritual : Essays in face to face behavior, Chicago, Aldine Transaction.

Gohard-RAdenkovic A. \& STALDER, P. (2013), « Pour en finir avec la conception ordinaire de la notion de culture... », Universitas 1, octobre, Fribourg, Université de Fribourg, 5354.

GrICE H. P. (1979), « Logique et conversation », Communications 30, 57-72.

GRINDSTED A. (1997), "Joking as a strategy in Spanish and Danish negotiations", in F. Bargiela-Chiappini \& S. Harris (eds), The Language of Business: An International Perspective, Edinburgh, Edinburgh University Press, 159-182.

Guibert N. Paquerot M., Roques O. (2002), L'humour en management : un essai de structuration du domaine, Congrès des IAE, Paris (cités dans Bottega, 2008).

GÜlich, E. (1986A), «Soûl n'est pas un mot très français. Procédés d'évaluation et de commentaire métadiscursif dans un corpus de conversations en 'situation de contact' », Cahiers de linguistique française 7, 231-258.

GÜLICH E. (1986B), «L'organisation conversationnelle des énoncés inachevés et de leur achèvement interactif en 'situation de contact' », DRLAV 34-35, 161-182.

GÜLICH E. \& KotsChI T. (1983), «Les marqueurs de la reformulation paraphrastique », Cahiers de Linguistique française 5, 305-351.

GuMPERZ J. J. (1982b), Discourse strategies, Cambridge, Cambridge University Press.

Hall E. T. (1959/1984), Le langage silencieux, Paris, Éditions du Seuil. 
House J. (2002A), «Englisch als lingua franca: eine Bedrohung für die europäische Mehrsprachigkeit?», in H. Barkowski \& R. Faistauer (Hrsg.), ... in Sachen Deutsch als Fremdsprache, Schneider Verlag Hohengehren, 62-72.

House J. (2002B), « Developing pragmatic competence in English as a lingua franca », in K. Knapp \& Ch. Meierkord (eds), Lingua Franca Communication, Frankfurt am Main, Peter Lang, 245-267.

House J. \& Rehbein J. (2004), Multilingual Communication, Amsterdam/Philadelphia, John Benjamins.

JodelET D. (1997), Les représentations sociales, Paris, PUF.

KeCSKEs I. (2013), Intercultural Pragmatics, Oxford, Oxford University Press.

KECSKES I. (ce volume), Is research in intercultural communication changing the way we understand language?.

KeRBRAT-ORECCHIONI C. (1989), «Théorie des faces et analyse conversationnelle », J. Isaac (éd.), Le parler frais d'Erving Goffman, Paris, Éditions de Minuit.

KeRBRAT-ORECCHIONI C. (1990), Les interactions verbales 1, Paris, Armand Colin.

Kerbrat-OrecChioni C. (1992), Les interactions verbales 2, Paris, Armand Colin.

Kerbrat-OrecChioni C. (1996), La conversation, Paris, Seuil (Mémo).

KERBRAT-ORECCHIONI C. (1998A), Les interactions verbales 3. Variations culturelles et échanges rituels, Paris, Armand Colin/Masson.

KeRbrat-Orecchioni C. (1998B), «La notion de «négociation» en analyse des conversations. L'exemple des négociations d'identité», Revue de Sémantique et Pragmatique 3, 13-33.

Kilani M. (2009), Anthropologie, du local au global, Paris, Armand Colin.

Kotschi T. (1986), "Procédés d'évaluation et de commentaire métadiscursif comme stratégies interactives », Cahiers de linguistique française 7, 207-230.

LysGaARD S. (1955), "Adjustment in a Foreign Society: Norwegian Fulbright Grantees Visiting the United States", International Scoial Science Bulletin 7, 45-51.

LyTTLE J. (2007), “The judicious use and management of humor in the workplace", Business Horizons (2007) 50, 239-245.

MaAlouf A. (1998), Les identités meurtrières, Paris, Editions Grasset \& Fasquelle.

MAHAdEVAN J. \& KILIAN-YASIN K. (2013), «Interkulturelles Lernen im berufsbezogenen Kontext », in K. v. Helmolt, G. Berkenbusch \& W. Jia (Hrsg.), Interkulturelle Lernsettings. Konzepte-Formate-Verfahren, Stuttgart, Ibidem-Verlag, 151-174.

Martin D. M., Rich, C. O., \& GAYLE, B. M. (2004), "Humor works: Communication style and humor functions in manager/subordinate relationships", Southern Communication Journal, 69, 206-222.

Martineau W. H. (1972), "A model of the social functions of humor", in J. Goldstein \& P. McGhee (eds.), The Psychology of Humor, New York, Academic Press, 101-125.

MERKIN R. S. (2018), Saving Face in Business. Managing Cross-Cultural Interactions, New York, Palgrave Macmillan.

Morreall J. (1989), "Enjoying incongruity, Humor", International Journal of Humor Research 2(1), 1-18.

Moscovici S. (1986), «L'ère des représentations sociales », in W. Doise (éd.), L'étude des représentations sociales, Neuchâtel, Delachaux et Niestlé, 34-80.

Mutabazi E. \& Pierre, Ph. (2008), Pour un management interculturel. De la diversité à la reconnaissance en entreprise, Paris, L'Harmattan. 
Neuiliep J. W. (2009, 4th ed.), Intercultural Communication : A Contextual Approach, Los Angeles, Sage.

PluTA P. (2014), "Understanding Humour in a training and development concept - the Diversity Icebreaker", Annual Conference of the International Society for Humor Studies (ISHS), Utrecht, (unpublished conference paper).

PluTA P. (2015), "Systematic use of humour in HR training concepts - an example of the Diversity Icebreaker", Paper presented at the 23rd Nordic Academy of Management Conference NFF - Business in Society, Copenhagen, Copenhagen Business School, 12-14 August, 1-27.

Pluta P. \& HöBER A. (2017), "Influence of individual differences in humor use on work engagement", Paper presented the 24th Nordic Academy of Management Conference NFF - Nordic Opportunities, Bodø, Nord University Business School, 23-25 August, 1-43.

PORCHER L. (2002), «L'humour comme le tango : une pensée triste qui se danse... »Le français dans le monde $\mathrm{n}^{\circ}$ spécial, 48-53.

Rivera A. (2000), «Culture », in R. Gallissot, M. Kilani \& A. Rivera, L'imbroglio ethnique, Lausanne, Payot, 63-82.

STAlder P. (2010), Pratiques imaginées et images des pratiques plurilingues. Stratégies de communication dans les réunions en milieu professionnel international, Berne, Peter Lang.

STALDER P. (2012), «Observation, écriture et décripture: radiographie d'un journal de terrain », in A. Gohard-Radenkovic, S. Pouliot \& P. Stalder (éds), Journal de bord, journal d'observation. Un récit en soi ou les traces d'un cheminement réflexif, Berne, Peter Lang, 49-72.

STALDER P. (2013), « Management international: développer les compétences en innovant les formations », in X. Yang \& L. Zheng, Culture et management, Paris, L'Harmattan, 215223.

STALDER P. (2014A), «La médiation plastique pour le développement des compétences de communication en milieu professionnel international : Compad®, Playmobils® \& Cie », in P. Stalder \& A. Tonti (éds), La médiation interculturelle : représentations, mises en œuvre et développement des compétences, Paris, Editions des archives contemporaines, $177-195$.

STALDER P. (2014B), « Strike Season? Gestion des diversités linguistiques et culturelles dans les réunions en milieu professionnel international. Recherches en didactique des langues et des cultures » Les Cahiers de l'Acedle 11(2), Plurilinguisme(s) et entreprise : enjeux didactiques, 11-32 [accédé le 24-09-20, http://acedle.org/old/IMG/pdf/02_Stalder_RDLC_v11n2.pdf].

StAlder P. (2016A), « Berne, Bucarest, Delphes. Aperçu d'une hybridation de démarches et méthodes pour appréhender le lien entre la diversité et l'innovation en Suisse », Recherches qualitatives, Hors-série 20, 416-432 [accédé le 24-09-20, http://www.recherche-qualitative.qc.ca/documents/files/revue/hors_serie/HS-20/rq-hs-20stalder.pdf].

STALDER P. (2016B), « 'J'existe, car je me souviens'. La réflexivité au profit du management interculturel », in P. Banon \& C. Blons-Pierre (éds), Didactique du français langue étrangère et seconde dans une perspective plurilingue et pluriculturelle, Berne, Peter Lang, 241-257.

STALDER P. (2018), « Management interculturel : entre théorie et anarchie », in P. R. Cloet, A. M. Guénette, E. Mutabazi \& Ph. Pierre (éds), Le défi interculturel. Enjeux et perspectives pour entreprendre, Paris, L'Harmattan, 141-149. 
STALder P. (2019A), «Funo ? Humour, émotion et créativité au sein d'équipes interculturelles », in F. Berdal-Masuy (éd.), Emo-langages: Vers une approche transversale des langages dans leurs dynamiques émotionnelles et créatives, Revue TIPA (Travaux interdisciplinaires sur la parole et le langage) 35, [en ligne, http://journals.openedition.org/tipa/3260 ; DOI : 10.4000/tipa.3260]

STALDER P. (2019B), "Creativity in Intercultural Teams: Which Indicators, Facilitators and Competencies?", IACCM-IESEG Conference Proceedings, Intercultural competencies for a disruptive VUCA world: Exploring creativity, innovation, resilience \& resistance in intercultural research, training \& management”, Paris, IÉSEG School of Management 31.10.-2.11.19, 9-27.

Stoiciu G. (2011), « La communication interculturelle comme champ d'études. Histoire, carte et territoire », in C. Agbobli et G. Hsab (éds), Communication internationale et communication interculturelle. Regards épistémologiques et espaces de pratique, Montréal, Presses de l'Université du Québec, 46-70.

Stoiciu G. \& HSAB G (2011), «Communication internationale et communication interculturelle : des champ croisés, des frontières ambulantes », in C. Agbobli \& G. Hsab (éds), Communication internationale et communication interculturelle. Regards épistémologiques et espaces de pratique, Montréal, Presses de l'Université du Québec, $10-25$.

Ting-ToOmeY S. \& Kurogi A. (1998), "Facework competence in intercultural conflict: An updated face-negotiation theory", International Journal of Intercultural Relations 22(2), $187-225$.

TRICART CH. (2015), L'humour au service de la performance. Riez et réussissez !, Genève, Jouvence.

Watzlawick P. (1984), Faites vous-mêmes votre propre malheur, Paris, éditions du Seuil.

WiCKER H.-R. (1997), "From complex culture to cultural complexity", in P. Werbner \& T. Modood (eds), Debating Cultural Hybridity, Multi-Cultural Identities and the Politics of Anti-Racism, London, Zed Books, 29-45.

WinkIN Y. (2001), Anthropologie de la communication, Paris, Seuil.

Wittezaele J. J. \& Garcia, T. (2002), A la recherche de l'école de Palo Alto, Paris, Editions du Seuil.

Wood R. E. Beckmann, N. AND Rossiter, J. R. (2011), "Management humor: asset or liability?", Organizational psychology review 1 (4), 316-338. 